\title{
Effects of Craniofacial Structures on Mouse Palatal Closure In Vitro
}

\author{
L. BRinkley, G. BASEhoar, and J. AVERY \\ Department of Oral Biology, School of Dentistry, The University of Michigan, Ann \\ Arbor, Michigan 48109, USA
}

Heads of Swiss-Webster mouse fetuses of four ages spanning days 12-13 of gestation, were partially dissected by removing the brain $(B)$, tongue $(T)$ and mandible $(M)$ alone or in combination $(B T, B M, B T M)$. Preparations were suspended in a gassed, circulating culture system such that palatal closure must take place against gravity. Closure occurred earlier than in vivo and required the posterior half of the mandible be intact and the tongue removed.

\section{J Dent Res 57(2): 402-411, February 1978.}

Although the development of the secondary palate has been the subject of extensive research in vivo and in vitro, little direct experimentation on the consequences of removing or modifying various craniofacial structures has been performed due to technical problems. Palatal closure, elevation and fusion involve many coordinated events, ${ }^{1}$ but the morphological capabilities of the shelves themselves and their interactions with other craniofacial structures have not been fully defined. Manipulation of the tongue in living fetuses around the time of palatal elevation has been the primary experimental operation to date. ${ }^{2-4}$

Formation of the secondary palate involves movement of the palatal shelves from a position lateral to the tongue to a horizontal plane above it. Subsequently the shelves fuse with each other at their medial edge and with the overlying nasal septum to complete palatal closure. The mechan-

\footnotetext{
Received for publication June 7, 1977.

Accepted for publication September 29, 1977

This work was supported by USPHS Grant DE-02774 from the National Institutes of Health, Bethesda, Maryland.

Portions of this paper were presented at the 52nd general meeting of the International Association for Dental Research, held in Atlanta, Georgia, March, 1974.
}

ism of shelf transposition from vertical to horizontal remains unknown despite much research on the problem. The palatal shelves have been shown to gain an "internal shelf force" of an undetermined nature $^{2}$ which permits at least some transposition. Additionally, several factors external to the palate including tongue displacement, ${ }^{3}$ straightening of the cranial base, ${ }^{5}$ and nonpalatal muscular activity such as mouth opening, swallowing and neck flexion, ${ }^{6.7}$ have also been implicated in palatal closure.

Previous in vitro experiments on palatal elevation have utilized only the middle one-third of the fetal face and have not attempted to alter experimentally the structures in the oral cavity. ${ }^{8-10}$ It is theoretically possible, given the appropriate culture system, to surgically remove a variety of craniofacial structures and observe the effects on palatal closure. Such studies could provide information on the developmental capabilities of the palatal shelves themselves and on the range of their activities in the presence and absence of certain anatomical features. We have previously reported on an in vitro system in which palatal closure occurs in partially dissected fetal mouse heads. " During the course of devising this system two types of dissected preparations were used: tongue and brain removed, and tongue, brain and mandible removed. It was found that heads with the tongue and brain removed always made much greater progress toward palatal closure than did those with the tongue, brain and mandible removed.

The objective of the present study was to expand our initial experiments to determine what craniofacial structures must be present or absent for closure to take place 
in vitro. We have studied the effects of removal of the brain, tongue and mandible, alone or in combination, on palatal shelf elevation in embryos of four developmental ages.

\section{Materials and Methods}

ANimals. - Timed-pregnant SwissWebster mice were obtained from Spartan Research (E. Lansing, Michigan). Fertilization was assumed to occur at $2 \mathrm{AM}$ of the day the vaginal plug was found (day 0 ). Procedures for obtaining and staging the fetuses have been described. ${ }^{11}$ Fetuses of four developmental ages were used. Their gestational ages, morphological ratings and approximate number of hours before expected in vivo closure are shown in Table 1. Morphological ratings (MR) were assigned by giving a numerical value to the developmental condition of forefeet, hindfeet, ears, hair follicles and eyes as described by Walker and Crain. ${ }^{12}$ Palatal closure in the mice used in these experiments occurs by MR 12 (14 day, 8 to 16 hours gestation).

Some variation in the developmental stage of littermates was found at all four ages used. Litters of younger fetuses (MR -2 to 0 and MR 1-2) showed little variation, while those of MR 3.4 and MR 5-6 were more heterogeneous in their development. Assignment of a morphological rating to a litter was based on the characteristics of the majority of fetuses. Those markedly advanced or retarded from the norm were discarded.

DisseCTIONS. - One fetus of a litter was fixed immediately and used to determine the state of the palate at the outset of culture. Littermates of the first three ages were divided into six groups. Each group was dissected in one of the following ways:

TABLE 1

Developmental Stages of Fetuses

\begin{tabular}{ccc}
\hline $\begin{array}{c}\text { Morphological } \\
\text { Rating }\end{array}$ & Gestational Age & $\begin{array}{c}\text { Hours before } \\
\text { closure }\end{array}$ \\
\hline$-2-0$ & 12d. $4-10 \mathrm{~h}$ & 52 \\
$1-2$ & 12d. 12-18h & 48 \\
$3-4$ & $13 \mathrm{~d} .4-10 \mathrm{~h}$ & 32 \\
$5-6$ & $13 \mathrm{~d} .8-14 \mathrm{~h}$ & 24 \\
\hline
\end{tabular}

tongue removed $(T)$, brain removed $(B)$, brain and tongue removed (BT), brain and mandible removed (BM), brain, tongue and mandible removed (BTM). To remove the brain, a single circumferential cut was made just above the eyes and the brain removed with forceps. When the tongue was removed but the oral cavity otherwise left intact ( $T$ and $B T$ ) a vent to the outside was cut by removing most of the soft tissues constituting the floor of the oral cavity while leaving the mandibular arch intact. This vent allowed greater medium circulation in the oral cavity. In BM preparations care was taken to leave the tongue undisturbed between the palatal shelves when removing a portion of the mandible. Approximately the anterior one-half of the mandibular tissues was removed. In this dissection the tongue and all mandibular tissue posterior to its root remained intact. $\mathrm{T}, \mathrm{B}, \mathrm{BT}$, and BTM dissections were performed on fetuses of the older age, but no $\mathrm{BM}$ dissections were done. None of the fetuses of any age showed spontaneous palatal shelf movement when the tongue was removed during dissection.

CULTURE. - Dissected preparations were submerged in the culture chamber by passing one end of an S-shaped stainless steel hook through the foramen magnum, and the other over wires fixed in the chamber. Each head was suspended with the nose down in the slightly turbulent medium such that palatal closure would have to be accomplished against gravity. This was carried out to better assess the dynamic forces of palatal closure. The culture medium was continually circulated with a peristaltic pump and superoxygenated to $\mathrm{pO}_{2}$ $430-460 \mathrm{~mm}$ using hollow fiber devices. Preparations were cultured at $34 \mathrm{C}$. Culture medium containing supplemented Eagle's basal medium and $50 \%$ heat inactivated fetal calf serum was used. The culture chamber, medium and culture conditions are reported in detail elsewhere. ${ }^{11}$ Five hundred sixty-two preparations were cultured 24 hours; 84 others were cultured for shorter periods as detailed in the results section. Uncultured heads and cultured preparations selected to represent all dissections were either fixed in Bouin's fixative, embedded in Paraplast, sectioned at 

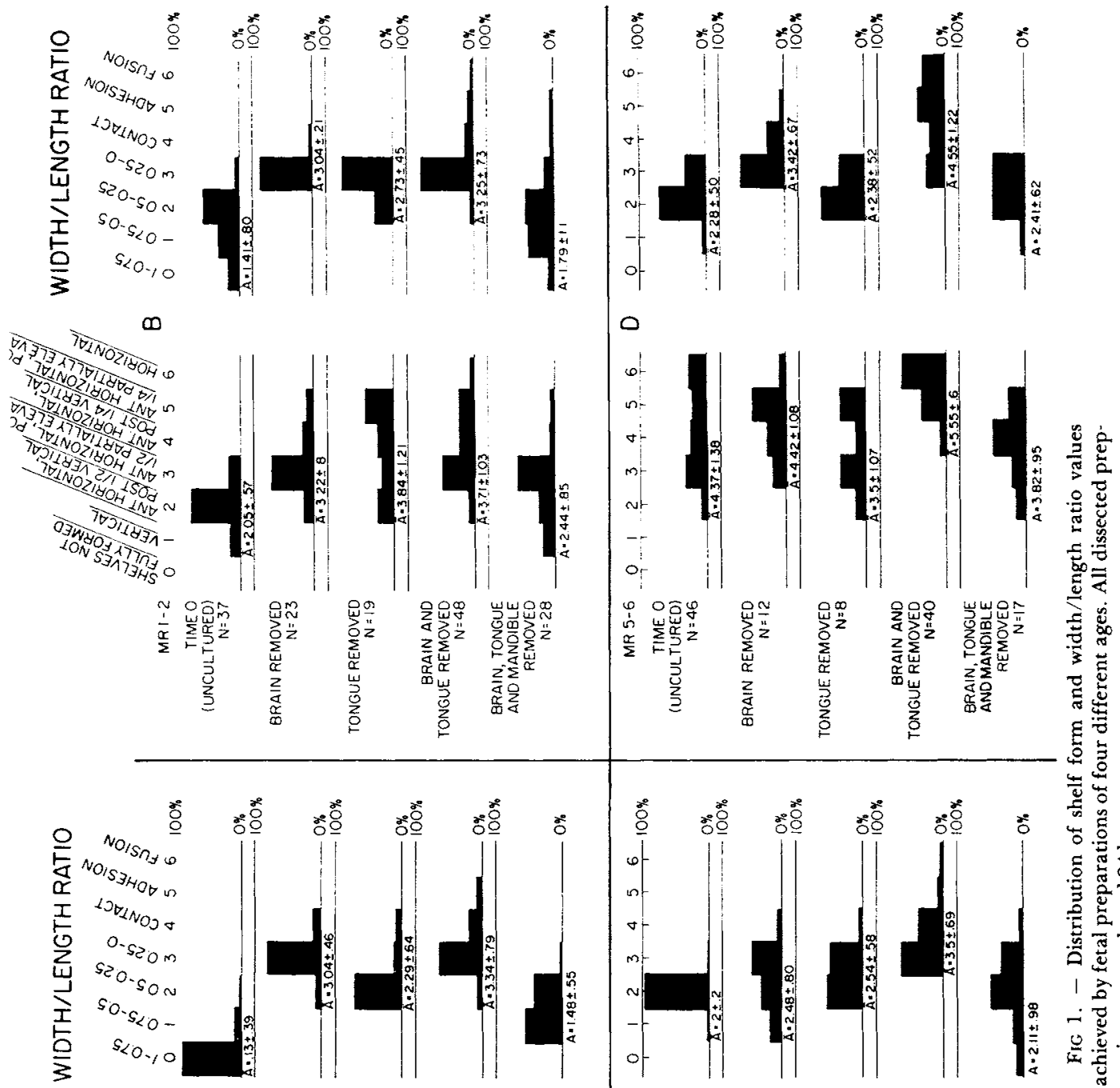

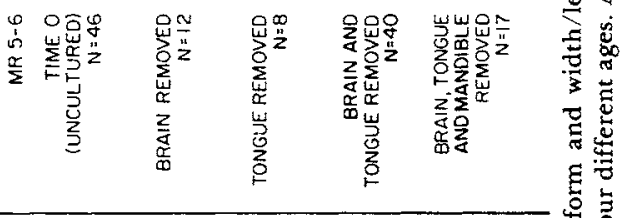

$\stackrel{5}{0}$
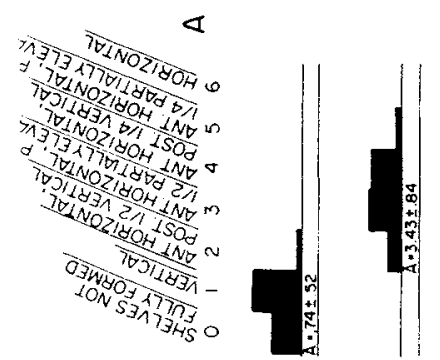

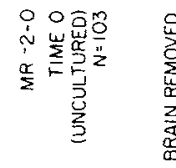

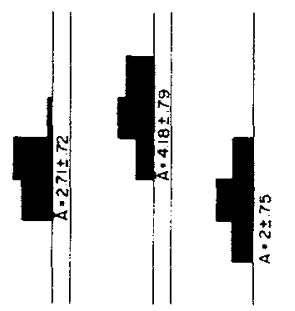

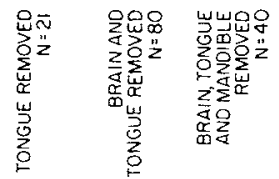
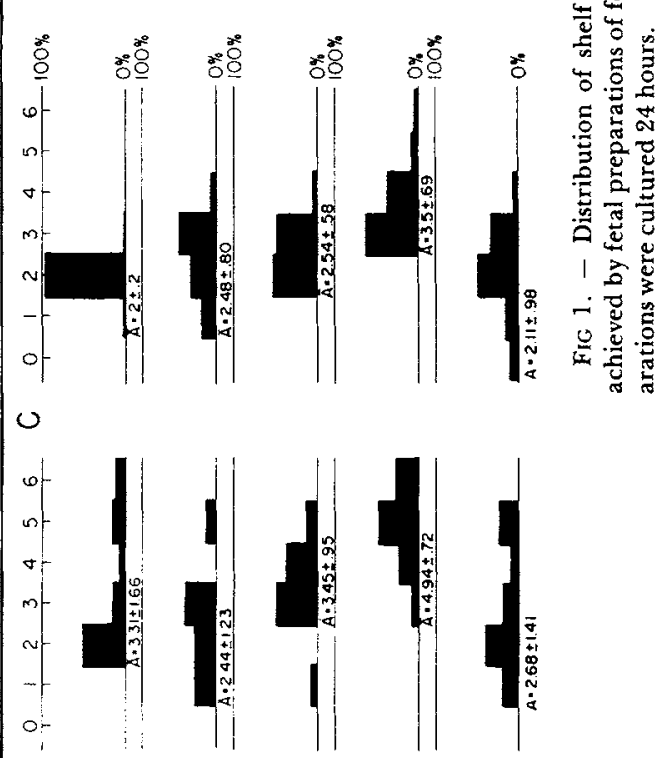

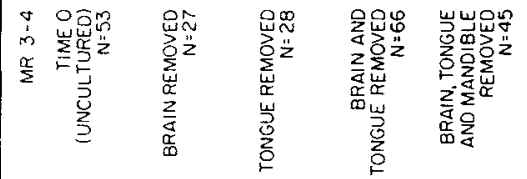


10 microns and stained with hematoxylin and eosin; or fixed in phosphate buffered formalin, embedded in methacrylate and sectioned at 3 to 5 microns.

RATING OF ELEVATION. - To allow statistical comparisons between experimental groups a numerical system of rating palatal closure was devised. Both fixed uncultured heads and their cultured littermates were examined using a dissecting microscope and values were assigned for both shelf form and width/length ratio. The gap between the shelves was measured with an ocular micrometer and the ratio of the width of the gap at the closest point to the length of the shelves was determined. This is described as the "width/length ratio" (Table 2). ${ }^{11}$

STATISTICS. - An analysis of variance was done on the mean shelf form ratings and width/length ratios of different dissections of fetuses of the same age, and also on the ratings of a given dissection done on fetuses of different ages. Differences were tested for significance using Scheffé's methods of multiple comparison. ${ }^{13}$

\section{Results}

Two variables were considered in these studies: the type of dissection and the developmental age at the outset of culture.
Figure 1 displays the distribution of shelf form ratings and width/length ratio values of both uncultured (time 0 ) and dissected, cultured fetal heads of the four ages studied. The mean and standard deviation for each set of values is given beneath the corresponding histogram. These values were used to determine statistical differences by a pairwise analysis of variance as described in the Materials and Methods.

INFLUENCE OF DISSECTION ON PALATAL CLOSURE. - All four types of dissected preparations of fetuses of the youngest age, MR -2-0 made progress toward palatal closure. Removal of the brain and tongue (BT) resulted in both the highest shelf form values and the most advanced width/ length ratios of the four types of preparations studied (Figs $1 \mathrm{~A}$ and 2 ); while removal of the brain, tongue and mandible (BTM) resulted in the least progress by both measures of palatal closure. A scale of progress of $\mathrm{BT}>\mathrm{B}>\mathrm{T}>\mathrm{BTM}>$ uncultured (time 0 ), for both shelf form and width/length ratio was observed for preparations of MR -2-0. Differences between all possible pairwise combinations of dissections were found to be significant ( $P=$ 0.01 ).

Removing the brain and tongue also resulted in the best progress at MR 3-4 and 5-6 (Figs 1C and D). However, at MR 1-2

TABLE 2

Numerical System of Rating Palatal Closure

\begin{tabular}{|c|c|c|}
\hline $\begin{array}{l}\text { Value } \\
\text { Assigned }\end{array}$ & Shelf Form & Width/length ratio \\
\hline 0 & $\begin{array}{l}\text { Uniform thin ridges of tissue, shelves } \\
\text { not fully formed } \ldots \ldots \ldots \ldots \ldots\end{array}$ & $1-0.75$ \\
\hline 1 & Vertical along entire length & $0.75-0.5$ \\
\hline 2 & $\begin{array}{l}\text { Anterior horizontal, posterior } \\
\qquad 1 / 2 \text { vertical } \ldots \ldots \ldots \ldots \ldots \ldots\end{array}$ & $0.5-0.25$ \\
\hline 3 & $\begin{array}{c}\text { Anterior horizontal, posterior } 1 / 2 \\
\text { partially elevated } \ldots \ldots \ldots \ldots\end{array}$ & $0.25-0$ \\
\hline 4 & $\begin{array}{c}\text { Anterior horizontal, posterior } \\
1 / 4 \text { vertical . . . . . . . }\end{array}$ & Contact \\
\hline 5 & $\begin{array}{l}\text { Anterior horizontal, posterior } \\
1 / 4 \text { partially elevated } \ldots .\end{array}$ & Adhesion \\
\hline 6 & Fully horizontal along entire length. . & Fusion* \\
\hline
\end{tabular}

*Evidence of epithelial breakdown: shelves appear continuous under the dissecting microscope, or show epithelial breakdown histologically. 


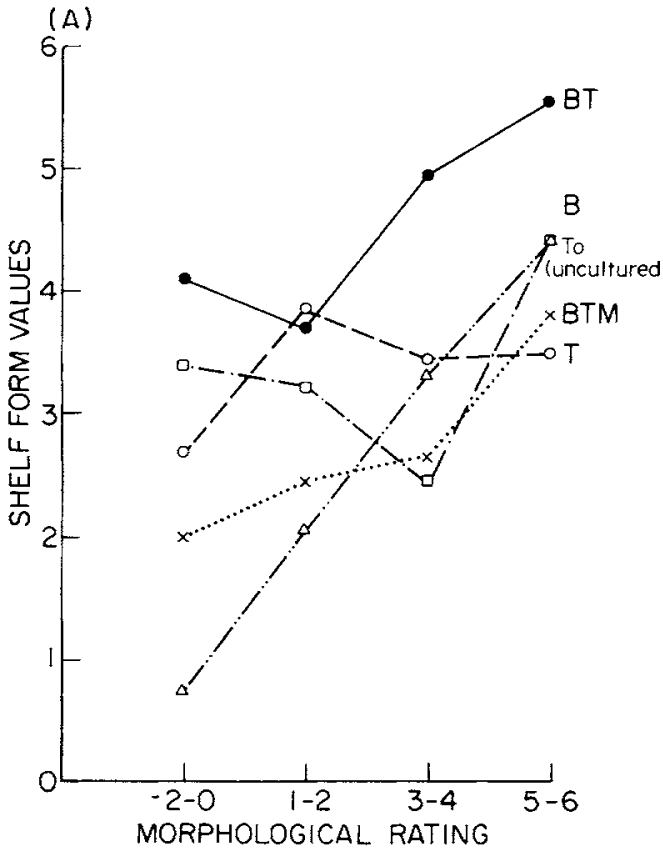

FIG 2. - Influence of developmental age on palatal closure in dissected fetal mouse heads. Mean values shown in Figure 2, are plotted from both (A) shelf form and (B) width/length ratio value, $\Delta=$ uncultured $\left(\mathrm{T}_{o}\right), \square=$ brain removed $(\mathrm{B}) ; \mathrm{C}=$ tongue $(\mathrm{T}) \mathrm{re}$ moved; - brain and tongue (BT) removed; $\mathrm{X}=$ brain, tongue and mandible (BTM) removed. Preparations described as different have $P=0.05$. (A) Shelf form: all dissections of MR -2.0 are different from one another, whereas at MR 1-2 only BT,

the removal of the tongue alone ( $T$ ) resulted in a mean shelf form that was statistically similar to that of $\mathrm{BT}$ preparations (Fig IB); although BT preparations attained significantly more advanced width/length ratios than did those with only the tongue removed $(P=0.01)$ (Fig 2$)$. Removing the brain (B) or the tongue (T) from fetal heads of MR 3-4 resulted in no change in shelf form. But tongue removal did result in a significant change in width/length ratios over that seen in the uncultured $\left(T_{0}\right)$ preparations (Figs $1 \mathrm{C}$ and 2).

Considering both shelf form and width/ length ratio, only removal of both brain and tongue consistently resulted in palatal closure. If the mandible was also removed (BTM), this result was negated. Removal of the brain alone or the tongue alone gave positive results at times.

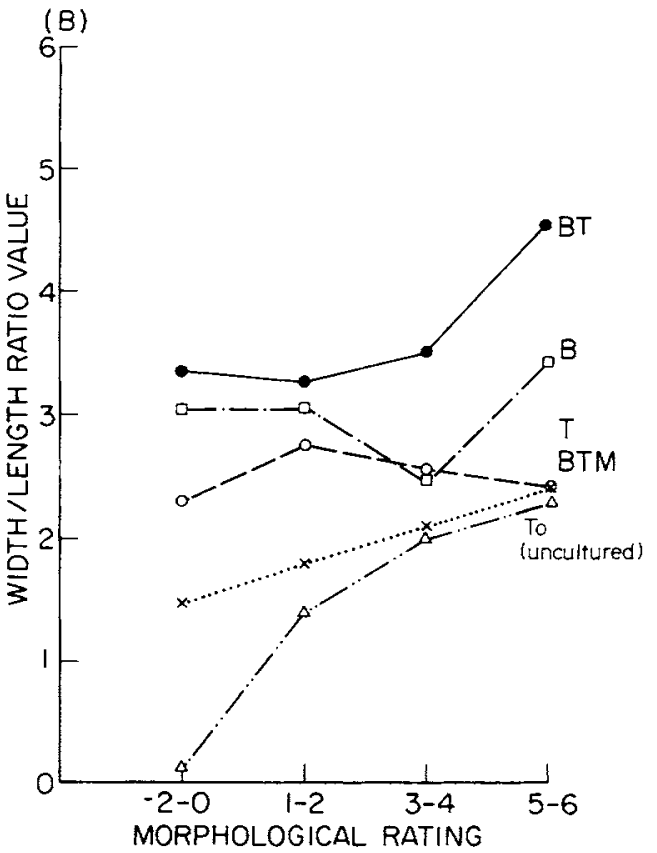

$\mathrm{B}$ and BTM differ significantly. Only BT makes significant progress from the starting point $\left(\mathrm{T}_{o}\right)$ at both MR 3-4 and MR 5-6. (B) Width/length ratio: All four types of dissections are different from one another and their $\mathrm{T}_{O}$ at both MR -2-0 and MR 1-2. At MR 3.4 BT differs from the remaining three dissections, while $T$ differs from uncultured preparations. Both BT and B are different from each other and the remaining preparations at MR 5-6.

INFLUENGE OF AGE ON PALATAL GLO. SURE. - The mean values for both shelf form and width/length ratio attained by each type of dissected preparations are plotted against developmental age at the outset of culture in Figure 2.

SHELF FORM. - BT preparations showed increasing progress in shelf form with age at three of the four ages used; at MR 1-2, BT also showed change after culture, however it was less than that seen at MR -2-0. Preparations with intact oral cavities (B) made good progress at the two youngest ages, MR $-2 \cdot 0$ and $M R 1-2$, but made no significant progress at the two older ages. When the tongue alone $(T)$ was removed advancement in shelf form was found at only the two youngest ages, with the highest mean value achieved at MR $1-2$. 
WIDTH/LENGTH RATIOS. - All four types of preparations of MR $-2-0$ and $1-2$ showed advancement in width/length ratios after culture, with $\mathrm{BT}>\mathrm{B}>\mathrm{T}>$ BTM > uncultured for both ages. BT preparations also showed increasing progress in $W / L$ ratio at $M R$ 3-4 and 5-6. Tongue removal also resulted in progress at MR 3-4, but not at MR 5-6. At the latter age, removing the brain facilitated change in the width/length ratio. Removing the brain, tongue and mandible resulted in no progress at MR 3-4 and 5-6.

RELATIONSHIP OF SHELF FORM AND WIDTH/LENGTH RATIO. - Considering only the most advanced stages of palatal closure, that is, shelf form rating 5 or 6 and shelves which are contacting, adhered or fused (Table 2), BT preparations clearly progress more rapidly than all other types of dissections at all ages. At MR -2-0, 17\% of heads with the brain and tongue removed and only $4 \%$ of $B$ preparations achieved these ratings. None of the other preparations did so. The percentage of $\mathrm{BT}$ preparations in this category fell to $10 \%$ at MR $1-2$, but only $3.5 \%$ of one other type of dissection, BTM, achieved this level. Heads with the brain and tongue removed advanced to shelf form 5 and 6 and were contacting, adhered or fused in $40.5 \%$ of the cases at MR 3-4 and 72.5\% at MR 5-6. At the latter age $32 \%$ of the preparations had begun to fuse.

INFLUENCE OF THE MANDIBLE. - As BT preparations were consistently found to

TABLE 3

COMPARISON OF MEAN SHELF FORM AND WIDTH/LENGTH. Ratio VAlues ATtained AfTer CUlture by DisSeCted PReParations With THE MANDible Partially OR COMPletely Removed

\begin{tabular}{|c|c|c|}
\hline & $\begin{array}{l}\text { Mean } \\
\text { Shelf Form Value }\end{array}$ & $\begin{array}{l}\text { Mean Width/ } \\
\text { length ratio value }\end{array}$ \\
\hline \multicolumn{3}{|l|}{ MR-2-0 } \\
\hline $\begin{array}{l}\text { Brain and tongue removed } \\
\qquad(\mathrm{n}=80) \ldots \ldots \ldots \ldots\end{array}$ & $4.18 \pm .79^{*}$ & $3.34 \pm .79^{*}$ \\
\hline $\begin{array}{l}\text { Brain and mandible removed } \\
\qquad(\mathrm{n}=15) \ldots \ldots \ldots \ldots\end{array}$ & $3.67 \pm .82$ & $2.67 \pm .73$ \\
\hline $\begin{array}{l}\text { Brain, tongue and mandible } \\
\quad \text { removed }(n=40) \ldots \ldots \ldots\end{array}$ & $2.0 \pm .75$ & $1.48 \pm .55$ \\
\hline \multicolumn{3}{|l|}{ MR 1.2 } \\
\hline $\begin{array}{l}\text { Brain and tongue removed } \\
\quad(n=48) \ldots \ldots \ldots \ldots\end{array}$ & $3.71 \pm 1.03$ & $3.25 \pm .73^{*}$ \\
\hline $\begin{array}{l}\text { Brain and mandible removed } \\
\qquad(\mathrm{n}=9) \ldots \ldots \ldots \ldots\end{array}$ & $4.33 \pm 1.0$ & $2.75 \pm .71$ \\
\hline $\begin{array}{l}\text { Brain, tongue and mandible } \\
\text { removed }(n=28) \ldots \ldots \ldots \ldots \ldots\end{array}$ & $2.44 \pm .85$ & $1.79 \pm 1.1$ \\
\hline \multicolumn{3}{|l|}{ MR 3-4 } \\
\hline $\begin{array}{l}\text { Brain and tongue removed } \\
\qquad(\mathrm{n}=66) \ldots \ldots \ldots \ldots \ldots \ldots\end{array}$ & $4.94 \pm \quad .85$ & $3.5 \pm .69$ \\
\hline $\begin{array}{l}\text { Brain and mandible removed } \\
\qquad(\mathrm{n}=12) \ldots \ldots \ldots \ldots\end{array}$ & $5.08 \pm 1.08$ & $3.83 \pm .72$ \\
\hline $\begin{array}{l}\text { Brain, tongue and mandible } \\
\text { removed }(n=45) \ldots \ldots\end{array}$ & $2.68 \pm 1.41$ & $2.11 \pm .98$ \\
\hline
\end{tabular}

Values attained by preparations with either the brain and tongue (BT) or the brain and mandible (BM) removed are statistically different from those achieved by heads with the brain, tongue and mandible removed for both shelf form and width/length ratio values at all three ages $(P \leq$ 0.01 ).

${ }^{*} \mathrm{BT}$ values are different from $\mathrm{BM}$ values with $P \leq 0.05$ 
make the most progress, and BTM usually the least, a variation of these dissections, removal of the brain and mandible (BM), was done on fetuses of three different ages. In these preparations mandibular tissue was removed from the corners of the mouth to the base of the tongue, leaving the tissue from that area posterior to the foramen magnum intact. At the end of the culture period for all ages studied, the tongue was always found to be displaced from between the shelves in BM preparations.

Table 3 contains the means and standard deviations for shelf form and width/ length ratios of BT, BM and BTM prepar-
A.

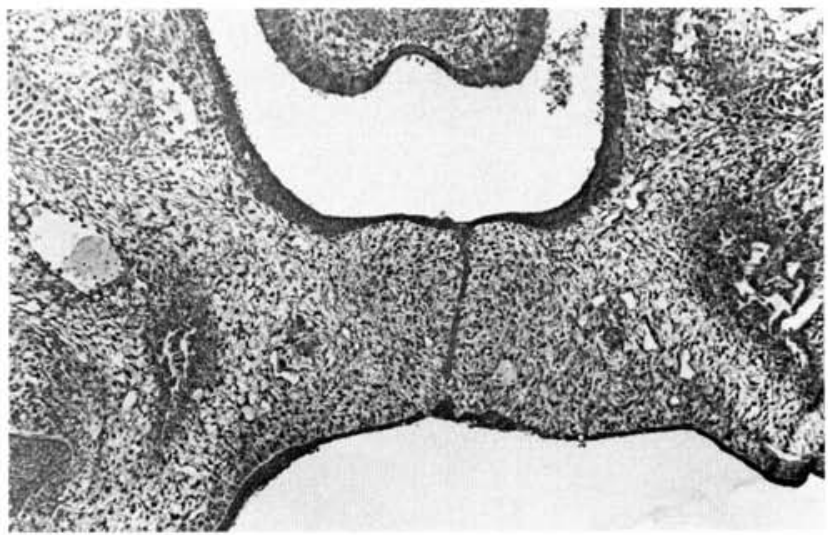

B.

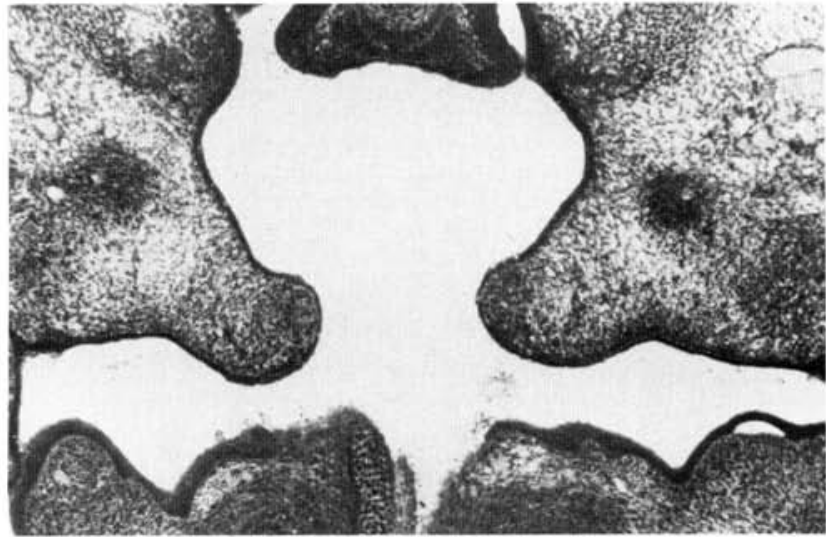

C.

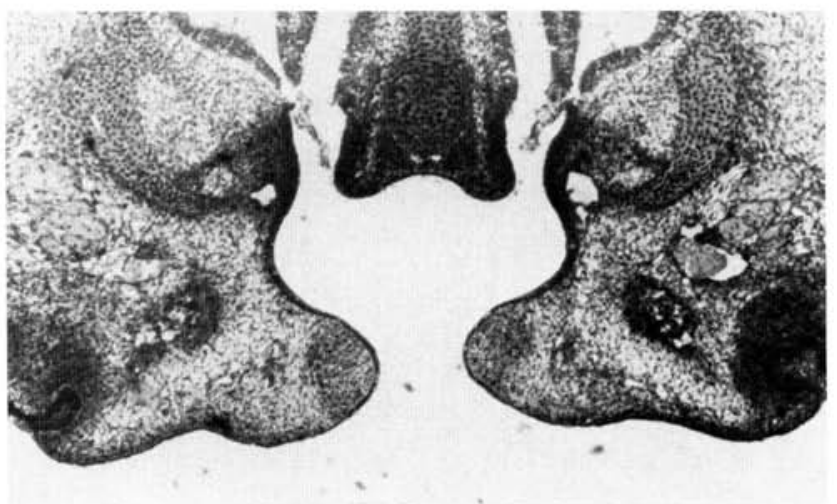

FIG 3. - Cross-section of palatal shelves of MR 3.4 fetal preparations cultured 16 hours, fixed, embedded in methacrylate, sectioned at $5 \mathrm{mi}$ crons and stained with toluidine blue. (A) Brain and tongue removed prior to culture. Palatal elevation has occurred and the shelves have adhered. The epithelial seam has been reduced to a single sheet and has begun to break down at two points (orig mag $125 \times$ ). (B) Tongue removed prior to culture. The shelves have not yet become fully horizontal (orig mag $80 \times$ ). (C) Brain, tongue and mandible removed prior to culture. Some elevation has occurred but a gap remains between the shelves. (orig mag 80 $x)$. 
ations for the three ages studied. BM heads were clearly more advanced than BTM for both parameters at all three ages. When shelf form alone is considered, BM preparations make good progress at all three ages, but BT are statistically more advanced at MR -2.0 $(P=0.05)$. The difference at MR 1-2 where $B M$ has a higher mean value than $B T$, is not significantly different, possibly due to the small sample size of BM. At MR 3-4 BM and BT are also similar.

CUlTuRE PERIOD. - Although the majority of preparations in these experiments were cultured for 24 hours, subsequent experiments with shorter culture periods were undertaken to determine the time required for palatal closure in vitro. Shortterm cultures ( 2 to 24 hours) of 84 fetal preparations with brain and tongue removed representing four different ages were conducted to determine how rapidly palatal closure occurs in vitro. Preparations that were taken 24 or 32 hours before in vivo closure required at least 4 hours' incubation before any form change was noticeable, whereas younger fetuses that were 48 to 52 hours prior to closure required about 6 hours to show a change in shelf form. Shelf form continued to change until about 12 to 16 hours of culture. From 16 to 24 hours of incubation no further major shelf form changes were seen, although width/length ratio changes did continue, principally moving from contact to fusion.

Histological findings indicated slight or no tissue degenerative change in preparations cultured less than 16 hours. Subsequently the condition of the tissue varied somewhat according to the type of dissection. Those with larger amounts of tissue present $(T)$ or with an intact oral cavity (B) showed more degenerative changes. This was probably due to poor medium circulation within the oral cavity. In those cases pycnotic nuclei and some detachment of the epithelial lining of the nasal cavity in the most interior area of these preparations were observed. The palatal shelves however, appeared to be in excellent condition in all types of preparations except those with the tongue in place (Fig 3). Although there was some variation among types of dissections in the vitality of the tissue, no direct connection between vitality and progress toward palatal closure was observed. Both BT and BTM preparations showed good tissue vitality, yet BTM preparations made the least progress in all cases.

\section{Discussion}

We have shown here and in a previous study" that in our culture system, partially dissected fetal mouse heads undergo the normal sequence of palatal elevation, against gravity, followed by adhesion and fusion. Our present data demonstrate the progress made by the dissected preparations is a function of both the morphogenetic capabilities of the palatal shelves at a given age and their interplay with the modified craniofacial environment created by removing the brain, tongue and/or mandible prior to culture. Palatal shelves have been previously shown to possess an "internal shelf force"2.14.15 which can result in shelf movement when the tongue is displaced from between the shelves close to the time of expected closure. Palatal closure observed in the present study may in part be the result of the acquisition or maturation of the mechanism underlying this force. Closure occurred in vitro in fetuses of all four ages used in these studies given the most anatomically favorable conditions, that is the removal of the tongue and brain. The earliest fetuses used were ap. proximately 52 hours prior to in vivo closure, were cultured 24 hours and achieved some elevation. Thus, under the conditions of this study palatal shelf force was exhibited at least 28 hours earlier than seen in vivo. Our observations indicate that appropriate tissue removal may effect expression of the internal shelf force.

Culturing fetal heads of the four different ages with an intact oral cavity (B), an oral cavity with the tongue removed (B'T) or one with both the tongue and mandible removed (BTM) provided information on interactions of the palatal shelves with structures comprising the oral cavity. Although BT preparations made the most progress of these three dissections at all ages, fetuses with only the brain removed made good progress at the two earliest ages. The palatal shelves themselves are 
capable of effecting form change if there is enough room for the shelves to maneuver within the oral cavity. At MR -2-0 and MR 1-2 the tongue appears to be sufficiently small that it is not an insurmountable barrier to the shelves. Also the total architecture of the oral cavity must be such that there is sufficient space between the nasal septum and the tongue for the shelves to interpose. A difference was seen at 32 hours pri ur to in vivo closure (MR 3-4); B heads achieved only slight advances in width/length ratio and none in shelf form (Fig 3). At this stage, the shelves appeared restricted by the large tongue so that no changes in shelf form could occur. These results agree with those of Walker and Quarles $^{2}$ who have shown that in fetuses which are developmentally close to palatal closure the tongue has an inhibitory effect on the initiation of closure. Wragg et al. ${ }^{16}$ have also shown that tongue width increases during the 24-36 hours prior to closure in the rat; this is probably the case in mice. Our results also suggest that major changes in the spatial relations within the oral cavity occur between 48 (MR 1-2) and 32 hours (MR 3-4) prior to in vivo closure.

Fetal heads with only the tongue removed $(\mathrm{T})$ provide a situation in which there is no intraoral barrier to shelf elevation. These preparations differ from BT only by the presence of the brain, yet palatal closure does not occur at all at all ages as it does when both the brain and tongue are removed. $T$ preparations make good progress at both the youngest ages, however, at the two later ages (18 to 24 hours prior to closure), no changes in shelf form were observed, whereas BT preparations undergo elevation at all ages. Possibly at the later ages some interaction of the brain and cranial base restricts progress. For approximately 24 hours prior to and during normal in vivo palatal closure the flexed cartilagenous cranial base straightens. ${ }^{17,18}$ Several authors have suggested this straightening is necessary for shelf elevation. ${ }^{19}$ How this is accomplished and whether or not the brain is involved is unclear. If cranial base straightening is involved in palatal elevation, our results suggests that the brain need not be present for the event to occur, although apparently in this in vitro system, the presence of the brain in older preparations may block palatal elevation. Further investigations on the possible relationship of brain development to cranial base changes and palatal closure might help clarify the question.

Finally, it is of interest to note that the posterior mandible has a profound effect on palatal elevation. Palatal closure is blocked by the removal of the entire mandible but is unaffected by removal of the anterior half.

\section{Conclusions}

The use of a gassed, circulating culture system in which the palatal shelves of suspended fetal heads must accomplish closure against gravity, permits direct evaluation of the relative importance of various factors on palatal elevation. The present study represents a successful attempt to experimentally evaluate the influence of several craniofacial structures on palatal closure. Our results agree with those of previous investigators that the shelves play a direct and active role in their own elevation. But further, we have found that in vitro palatal closure can occur earlier than in vivo. Given appropriate tissue removal, the palatal shelves have the capacity to initiate closure 1 to 2 days before in vivo closure will occur. Present data also provide sup. port for the view that the architecture of the craniofacial region plays an important role in permitting the shelves to express their closure potential. In vitro palatal closure requires the presence of the posterior half of the mandible, and although the presence of the brain has little effect in development of young fetuses, its relation to the cranial base may be important in later stages.

\section{References}

1. Burdi, H.; Feingold, M.; Larsson, K.; LECK, I.; ZIMMERMAN, E.; and FRASER, F.: Etiology and Pathogenesis of Congenital Cleft Lip and Cleft Palate. An NIDR State of the Art Report, Teratology 6:255-268, 1972 .

2. WALker, B., and Fraser, F.: Closure of 
the Secondary Palate in Three Strains of Mice. J Embryol Exp Morphol 4:176-189, 1956

3. WAlker, B. and Quarles, J.: Palate Development in Mouse Foetuses After Tongue Removal, Arch Oral Biol 21:405-412, 1976.

4. ATNIP, R.: The Role of Surface Tension in Experimentally Induced Elevation of Embryonic Palatal Shelves, Anat Rec 145:308, 1963.

5. Verrusio, A.: A Mechanism for Closure of the Secondary Palate. Teratology, 3:17-20, 1970.

6. Humphrey, T.: The Relation Between Human Fetal Mouth Opening and Reflexes and Closure of the Palate, Am J Anat 125:317-344, 1969.

7. WALKER, B.: Correlation of Embryonic Movement with Palate Closure in Mice, Teratology 2:191-198, 1969.

8. REEVE, W.; PORTER, K.; and LEFKOWITZ, W.: In Vitro Closure of the Rat Palate, $J$ Dent Res 45:1375-1380, 1966.

9. THOMpson, J., and SCHWEISTHAL, M.: Study of Closure of the Embryonic Rat Palate In Vitro with the Effects of Certain Chemicals, J Dent Res 48:568-572, 1969.

10. Lahti, A.; ANTIla, A.; and SAXEN, L.: The Effect of Hydrocortisone on the Closure of the Palatal Shelves in Two Inbred Strains of Mice In Vivo and In Vitro, Teratology 9:37-42, 1972.
11. BRINKLEY, L.; BASEHOAR, G.; BRANCH, A.; and AVERY, J.: A New In Vitro System for Studying Secondary Palate Develop. ment, $J$ Embryol Exp Morph 34:485-495, 1975.

12. WALKer, B., and Crain, B.: Effects of Hypervitaminosis A on Palate Development in Two Strains of Mice, Am J Anat 107:49-58, 1960.

13. SCHEFFE, H.: A Method of Judging All Contrasts in the Analysis of Variance, Biometrika 40:87, 1953.

14. Lazzaro, C.: Sul meccanismo del chivsura del palato secondario, Monit Zool Ital 52:249-273, 1940 .

15. Ross, L., and WALkeR, B.: Movement of Palatine Shelves in Untreated and Teratogen-Treated Mouse Embryos, Am J Anat 121:509-522, 1967.

16. WRAGG, L.; DIEWERT, V.; and KLEIN, M.: Spatial Relations in the Oral Cavity and the Mechanism of Secondary Palate Closure in the Rat, Arch Oral Biol 17:683-690, 1972.

17. HARRIS, J.: Oligohydramnios and Cortisone-Induced Cleft Palate, Nature, Lond 203:533-534.

18. DIEWERT, V.: Graphic Reconstructions of Craniofacial Structures During Secondary Palate Development in Rats, Teratology, 14:291-314, 1976.

19. Greene, R., and Pratt, R.: Developmental Aspects of Secondary Palate Formation, $J$ Embryol Exp Morph 36:245-255, 1976. 